\title{
The SCExAO high contrast imager: transitioning from commissioning to science
}

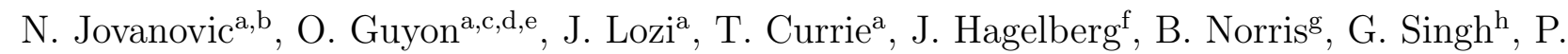
Pathak $^{\mathrm{a}}$, D. Doughty ${ }^{\mathrm{a}, \mathrm{d}}$, S. Goebel ${ }^{\mathrm{a}, \mathrm{f}}$, J. Males ${ }^{\mathrm{c}}$, J. Kuhn ${ }^{\mathrm{k}}$, E. Serabyn ${ }^{\mathrm{h}}$, P. Tuthill ${ }^{\mathrm{g}}$, G.

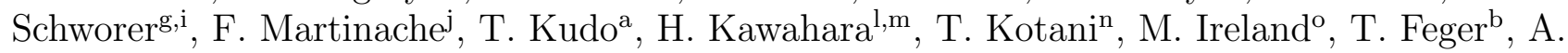

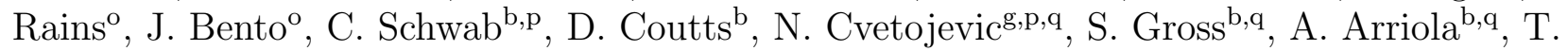

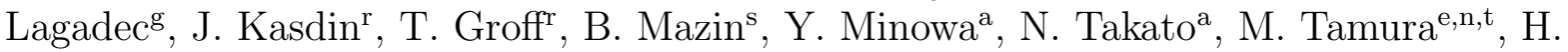
Takami $^{\mathrm{n}}$, and M. Hayashi ${ }^{\mathrm{n}}$

${ }^{a}$ National Astronomical Observatory of Japan, Subaru Telescope, Hilo, HI, 96720, U.S.A.

${ }^{b}$ Department of Physics and Astronomy, Macquarie University, NSW 2109, Australia ${ }^{\mathrm{c}}$ Steward Observatory, University of Arizona, Tucson, AZ, 85721, U.S.A.

${ }^{\mathrm{d}}$ College of Optical Sciences, University of Arizona, Tucson, AZ 85721, U.S.A.

${ }^{\mathrm{e}}$ Astrobiology Center of NINS, 2-21-1, Osawa, Mitaka, Tokyo, 181-8588, Japan

${ }^{\mathrm{f}}$ Institute for Astronomy, University of Hawaii, Honolulu, HI 96822, U.S.A.

${ }^{\mathrm{g}}$ Sydney Institute for Astronomy (SIfA), Institute for Photonics and Optical Science (IPOS), School of Physics, University of Sydney, NSW 2006, Australia

h Jet Propulsion Laboratory, 4800 Oak Grove Drive, MS 183-901, Pasadena, CA 91109

${ }^{\text {i}}$ LESIA, Observatoire de Paris, Meudon, 5 Place Jules Janssen, 92195, France.

jObservatoire de la Cote d'Azur, Boulevard de l'Observatoire, Nice, 06304, France

${ }^{k}$ Institute for Astronomy, ETH Zurich, Wolfgang-Pauli-Strasse 27, 8093 Zurich, Switzerland ${ }^{1}$ Department of Earth and Planetary Science, The University of Tokyo, Tokyo 113-0033, Japan ${ }^{m}$ Research Center for the Early Universe, School of Science, The University of Tokyo, Japan

${ }^{\mathrm{n}}$ National Astronomical Observatory of Japan, 2-21-1 Osawa, Mitaka, Japan

${ }^{\circ}$ Research School of Astronomy \& Astrophysics, Australian National University, Canberra ACT 2611, Australia

${ }^{\mathrm{p} A u s t r a l i a n}$ Astronomical Observatory, 105 Delhi Rd, North Ryde NSW 2113, Australia ${ }^{\mathrm{q}}$ Centre for Ultrahigh bandwidth Devices for Optical Systems (CUDOS)

${ }^{\mathrm{r}}$ Princeton University, Department of Mechanical and Aerospace Engineering, Engineering

Quadrangle, Olden Street, Princeton, New Jersey 08544, United States

${ }^{s}$ University of California, Department of Physics, Santa Barbara, California 93106, U.S.A.

${ }^{\mathrm{t}}$ The University of Tokyo, Tokyo 113-0033, Japan

\begin{abstract}
SCExAO is the premier high-contrast imaging platform for the Subaru Telescope. It offers high Strehl ratios at near-IR wavelengths ( $\mathrm{y}-\mathrm{K}$ band) with stable pointing and coronagraphs with extremely small inner working angles, optimized for imaging faint companions very close to the host. In the visible, it has several interferometric imagers which offer polarimetric and spectroscopic capabilities. A recent addition is the RHEA spectrograph enabling spatially resolved high resolution spectroscopy of the surfaces of giant stars, for example. New capabilities on the horizon include post-coronagraphic spectroscopy, spectral differential imaging, nulling interferometry as well as an integral field spectrograph and an MKID array. Here we present the new modules of SCExAO, give an overview of the current commissioning status of each of the modules and present preliminary results.
\end{abstract}

Nemanja Jovanovic: E-mail: jovanovic.nem@gmail.com, Telephone: 18089345959

Adaptive Optics Systems V, edited by Enrico Marchetti, Laird M. Close, Jean-Pierre Véran, Proc. of SPIE Vol. 9909,

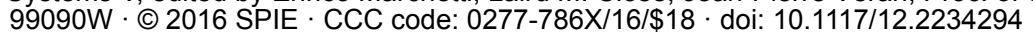


Keywords: Extreme AO, Adaptive optics, High contrast imaging, Exoplanets, Sparse aperture masking, Coronagraphs, Imager, Fiber injection

\section{INTRODUCTION}

High contrast imaging is the exciting field dedicated to directly imaging substellar companions around other stars. Key to detecting these companions is the ability to: 1) spatially separate the star from the orbiting object and 2) detect the faint object in the presence of the bright speckle background of the star. Both of these goals are aided by advanced adaptive optics (AO) systems known extreme AO (ExAO) systems. These systems are single conjugate $\mathrm{AO}$ systems which operate at higher temporal and spatial frequencies than conventional AO systems and can offer $\sim 90 \%$ Strehl ratios in the near-IR (H-band). This not only allows for the light from the host star and companion to be spatially separated to a large extent, but also improves the speckle dominated noise floor enabling the detection of faint companions.

There are a handful of AO systems now capable of reaching this level of performance in better than median seeing conditions, which includes GPI, ${ }^{1}$ SPHERE, ${ }^{2}$ PALM3000, ${ }^{3} \mathrm{MagAO}^{4}$ and LBTAO. ${ }^{5}$ The Subaru Coronagraphic Extreme Adaptive Optics (SCExAO) instrument is the latest addition to this suite. ${ }^{6}$ SCExAO is an instrument optimized for imaging and spectroscopic characterization at $<5 \lambda / D$ from the host star, a region the others can not easily access. In addition it is constantly evolving in order to incorporate the latest in state-of-the-art technologies. This makes it the ideal testbed for a high-contrast imager for an ELT.

In this paper we give an overview of SCExAO, including new modules, and outline the current commissioning status. We then go on to demonstrate the preliminary results achieved to date from science operation in regards to imaging disks, faint companions and dust shells around giant stars.

\section{INSTRUMENT OVERVIEW}

\subsection{The SCExAO instrument}

A schematic of the SCExAO instrument as of February 2016 is shown in Fig. 1. A comprehensive overview of the instrument can be found in Jovanovic et. al. (2015), so here we offer a basic overview and focus mainly on the new aspects of the instrument. In addition, we refer the reader for specific details and results about each module to the numerous other articles in the proceedings from this conference. ${ }^{7-15}$

SCExAO operates downstream of the facility AO system, AO188 ${ }^{16}$ at the Subaru Telescope. AO188 offers Strehl ratios of $30-40 \%$ in the H-band, in median seeing conditions. The corrected beam is injected into the bottom bench of SCExAO. After bouncing off of the 2000-element deformable mirror (DM, Bostom MicroMachines Corporation) the beam is split. Wavelengths shorter than $<950 \mathrm{~nm}$ are directed towards a periscope and routed to the visible bench. Longer wavelength light continues to propagate on the bottom bench (IR bench).

Light between $800-950 \mathrm{~nm}$ is fed to the pyramid wavefront sensor (PyWFS). This is the high order wavefront sensor SCExAO uses to reach ExAO levels of performance. This first stage of wavefront correction provided by AO188 is needed to make sure that the beam delivered to the PyWFS is within the linear range of the sensor. The PyWFS is used to drive the ExAO loop because of its exquisite sensitivity to high order modes and operates at $1.5-3.5 \mathrm{kHz}$ with very low latency $(\sim 1 \mathrm{~ms}))^{7,8}$ The left over light on the visible bench is used for scientific characterization by the VAMPIRES, FIRST and RHEA modules. VAMPIRES is an aperture masking inteferometer providing sub-diffraction limited spatial resolution images with polarimetry, and is commissioned and fully operational. ${ }^{12,17}$ FIRST is a pupil-remapping interferometer which provides spectral information in addition to high angular resolution images. ${ }^{13,14}$ It has not completed commissioning and is still undergoing further tests. RHEA, the newest addition is a high-resolution Echelle spectrograph with an integral field unit (IFU) for a feed (described in greater detail in Sec. 2.3). ${ }^{15}$

The light transmitted by the dichroic on the IR bench can be exploited for coronagraphy for example. In this case a number of coronagraphs including the Phase Induced Amplitude Apodization (PIAA) ${ }^{18}$ and the vector vortex $^{19,20}$ versions, to name a few. These coronagraphs are optimized for small inner working angle $(1-3 \lambda / D)$ operation in the near-IR (J-K bands). Post-coronagraph the light is fed to a facility imager. This is currently HiCIAO $^{21}$ and in the very near future, the integral field spectrograph, CHARIS. ${ }^{22,23}$

The following subsections summarize some of the recent upgrades to SCExAO. 


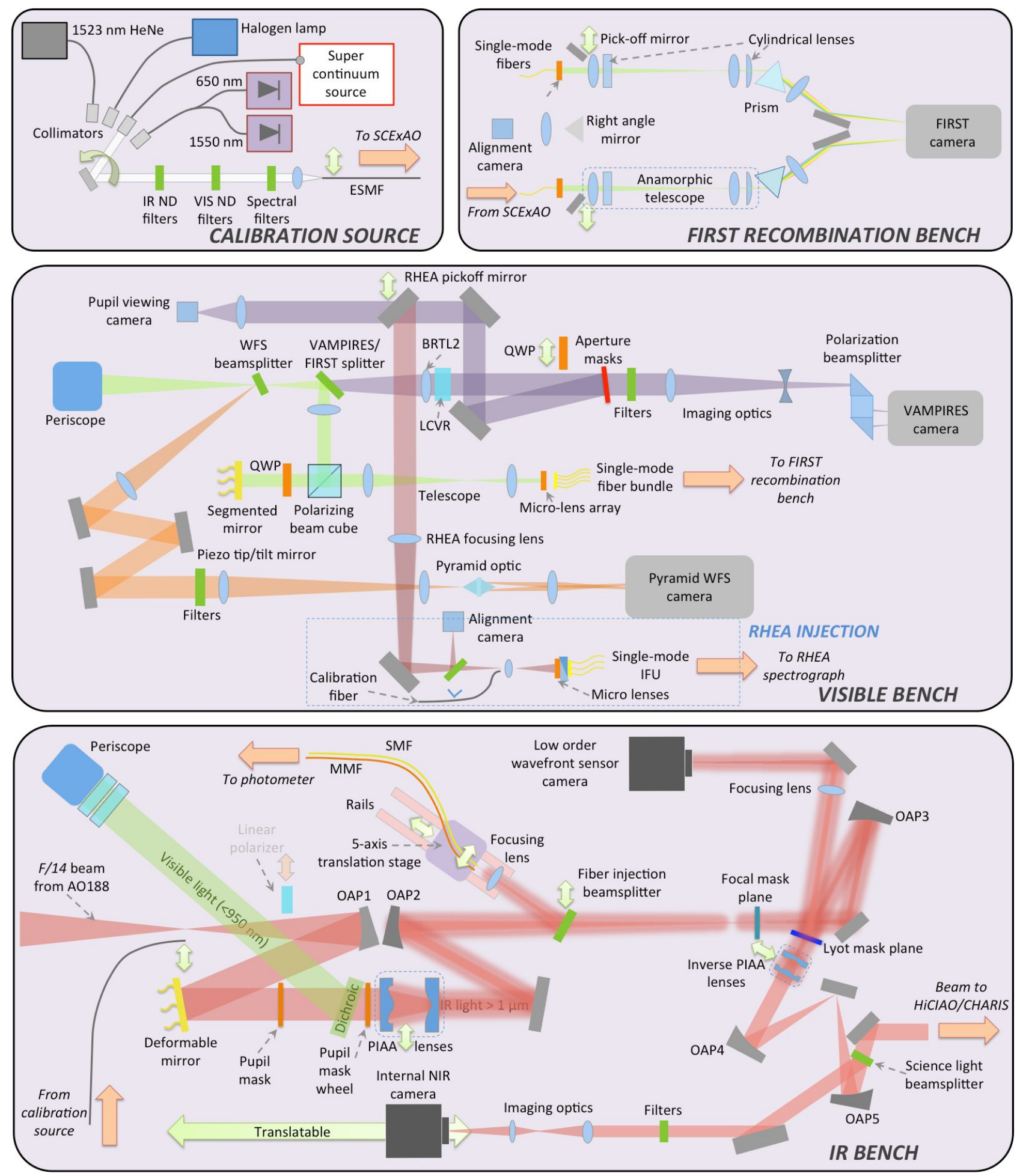

Figure 1. Schematic of the SCExAO instrument. (Top left) The calibration source which allows the user to select between a super continuum source, $1523 \mathrm{~nm}$ HeNe laser, 650/1550 nm laser diode and a halogen lamp. (Top right) The photometric setup for calibrating the coupling efficiency. (Middle) Visible bench of SCExAO which hosts the pyramid wavefront sensor. (Bottom) Infrared bench of SCExAO which hosts the fiber injection, the deformable mirror and the PIAA lenses. 

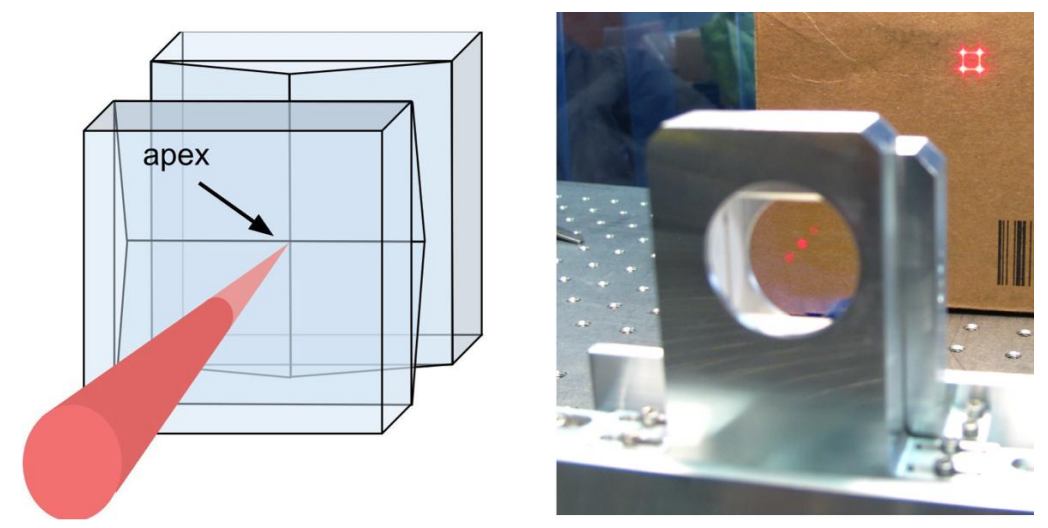

Figure 2. (Left) Schematic of the roof prism pyramid optic concept. (Right) Roof prism assembly diffracting a collimated HeNe beam into 4 beamlets.

\subsection{Roof-prism based PyWFS}

The pyramid optic in the primary wavefront sensor of SCExAO was replaced as it was only on loan. Obtaining a pyramidal optic is difficult. This is due to the fact that the four surfaces of the pyramid must meet at a single point, posing a serious challenge in polishing and that the tip of the pyramid must be sharp. A blunt tip will scatter/diffract light from the point-spread function (PSF) to regions of the pupil image that are not being used to measure the wavefront error or worse still, regions that are. In both cases this reduces the sensitivity of the wavefront sensor. For this reason we chose to build a PyWFS in a non-traditional approach. The concept utilizes two roof prisms oriented such that the roof vertices are orthogonal to one another (one runs vertical and the other horizontal) as shown in Fig. 2. The prisms are positioned such that the vertices are in close proximity to one another (spaced by $20-50 \mu \mathrm{m}$ ) and close to the focus of the beam. This means that the input beam enters through the flat polished bottom surface of the first roof prism and leaves through the corresponding surface of the second roof prism. By diffracting light from the vertex with a HeNe laser in the visible, the angle of the roofs was determined to be $1.867 \pm 0.107^{\circ}$. This shallow angle is relatively easy to realize for a roof prism, but compounds the difficulty in fabricating a pyramidal optic.

The new optics were used to replace the original pyramid in SCExAO in early 2016. The assembly can be seen in the right panel of Fig. 2 pre-installment in SCExAO. The PyWFS was realigned and has been subsequently tested. It has been confirmed that there is no noticeable difference in performance between the original pyramidal optic and the new roof prism concept. A more detailed characterization is needed to confirm this unequivocally.

In addition to the upgrades to the hardware, there have been numerous initiatives to improve the control software as well. ${ }^{7,8}$ This includes a move to using a zonal basis set of modes for the control matrix (modes defined by the DM actuators) as they are orthogonal in DM and wavefront sensor space (nearly). In addition, some low order Zernike modes, namely tip/tilt and focus are incorporated as it was determined that the zonal basis set did not offer a strong response to these modes. Numerous improvements to the computation speed have also been made by way of using multiple GPUs, streaming pixels as they are collected by the camera to begin calculation rather than waiting for full frame to arrive and isolating process on various cores. This has resulted in a reduction of the latency to $<1 \mathrm{~ms}$ at a loop speed of $3.5 \mathrm{kHz}$. Most recently a linear, quadratic, Gaussian controller has been implemented to control the low order modes of the PyWFS control loop. The PyWFS is operational with an LQG, and in better than median seeing, the system is delivering Strehl ratios of 65-75\%. Optimization of the software and parameters is needed to improve performance to the ExAO level goal.

\subsection{The Replicable High-resolution Exoplanet and Astroseismology spectrograph}

The RHEA spectrograph is unique in that it is fed by a single-mode fiber IFU. The IFU consists of a $3 \times 3$ array of $1 \mathrm{~mm}$ lenslets feeding single-mode fibers in the visible range. The projected on-sky separation of the fibers is 16 mas. The 9 fibers are injected into an instrument which was optimized for operation from $600-800 \mathrm{~nm}$ at a resolving power of $\sim 60000$. RHEA is very small (see Fig. 3), occupying a footprint of less than $30 \mathrm{~cm}^{3}$, built from entirely off-the-shelf optics. The R2 Echelle grating and spectrograph as a whole are easily stabilized with 

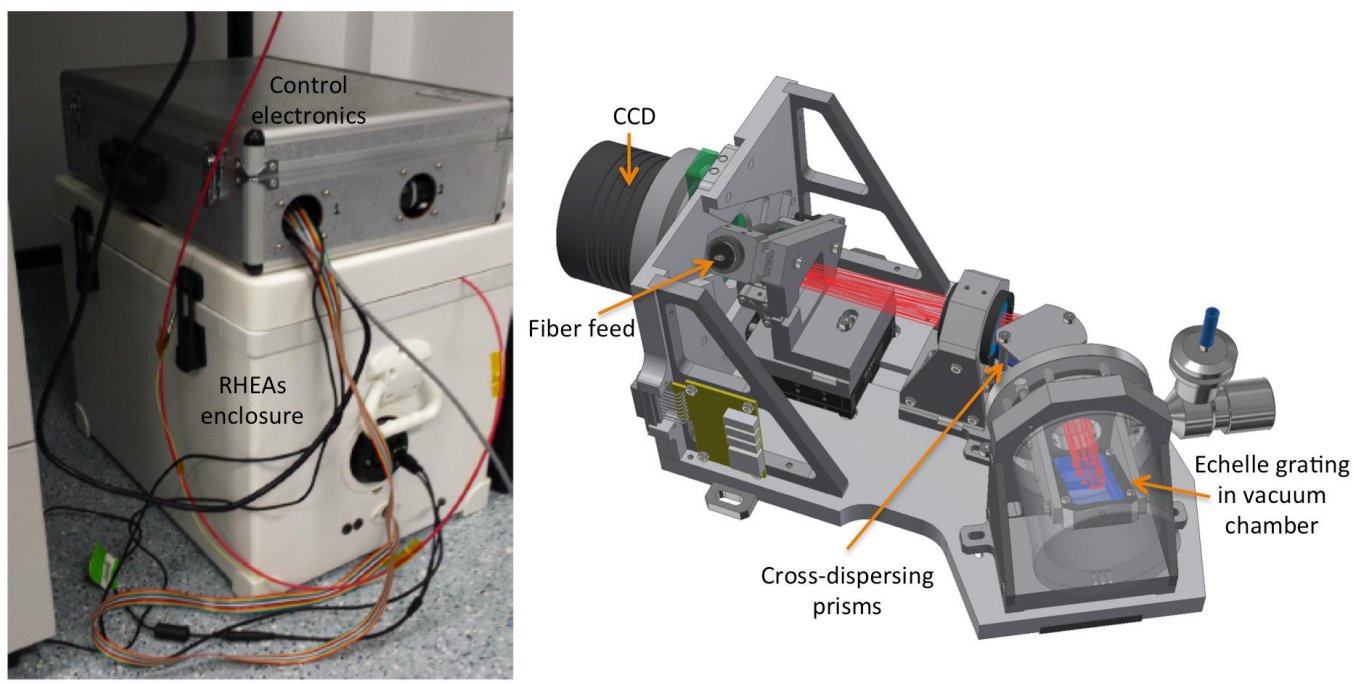

Figure 3. (Left) The RHEA spectrograph inside an icebox for thermal stabilization. Control electronics box can be seen on top. (Right) The RHEA spectrograph deployed at Subaru Telescope.

very simple temperature control. This means the instrument can achieve an intrinsic precision of $\sim 1 \mathrm{~m} / \mathrm{s}$ over a time period of days. ${ }^{24}$

The high spatial and spectral resolution make it ideal for mapping the surfaces of giant stars in order to study convection and stellar rotation for the first time, study resolved protoplanetary disk structures as well as for the detailed characterization of accreting protoplanets. ${ }^{25}$ An instrument like RHEA behind SCExAO could potentially shed light on the accretion rate and in-flow velocity, which would allow the mass and density of the forming exoplanet to be constrained. This data combined with the spatial location in the protoplanetary disk will aid in refining planetary formation models which are currently unconstrained.

The fiber injection for RHEA is located on the visible bench and shares the light with the VAMPIRES module. To center the IFU on a star, an alignment camera is used. This camera looks at the star and a beam which is counter-propagated from the spectrograph to the IFU and steered onto the camera via a retro-reflecting cube simultaneously. By translating the IFU laterally, its possible to overlap the reverse injected beam with the star which will ensure that the star is aligned with the IFU and will be coupled in. The injection and spectrograph have been installed on SCExAO and are currently undergoing commissioning. We aim to have this module transition to full science operation by $2017 .^{15}$

\subsection{The Calibration Source}

The calibration source has been upgraded so that now the user can remotely switch between light sources. As shown in Fig. 1 the calibration source box now hosts the super continuum source (Whitelase Micro, Fianium), the $650 / 1550 \mathrm{~nm}$ laser diodes combined by fiber splitter/coupler into a single fiber, a halogen lamp coupled into a single-mode fiber and a $1523 \mathrm{~nm}$ HeNe laser. These sources are collimated and then a mirror on a rotation stage is used to steer the beams into a collecting lens connected to a endlessely-single-mode fiber which routes the light to the SCExAO bench. Only one source can be injected at a time. The halogen lamp is used for flat fielding a test photonic spectrograph (not shown in the figure) as its spectrum is more stable than the super continuum source. All sources can be attenuated with the use of the neutral density filter wheels and the spectrum tailored as required. The calibration source now allows for the light to be remotely switched improving the speed and quality of calibration.

\section{RECENT RESULTS}

SCExAO is in a mixed state of commissioning and science operation. In this section we briefly outline some of the technical and scientific results to come from the instrument in the past year. 


\subsection{Advanced calibration speckles}

Artificial speckles are now commonly used on high contrast imagers like GPI and SPHERE to calibrate the photometry (contrast) and astrometry of the faint companion. Recently we have demonstrated that it is possible to improve the stability of the speckle grid, which is used as a ruler to determine the astrometry and photometry of the companion, by modulating the phase of the speckles by $180^{\circ}$ rapidly ${ }^{26}$ during an exposure. The modulation in phase means that the artificial speckle will coherently add in one instance and subtract in another, with the speckle background. If the modulation is fast enough that the speckle background is static over that time frame, and an exposure is taken during this period, then the resulting image is the sum of the two speckle fields which yields an image where the artificial speckle is incoherently summed with the speckle background. This is extremely important as the speckle is therefore not modulated by the background and retains its intended contrast and centroid location. In this way a speckle grid immune to perturbations by the background speckle field can be generated which will be very important for advanced high contrast imagers. The preliminary work showed a factor of 2-3× improvement in the stability of the grid in contrast and location ${ }^{26}$ for only 100 modulations per second. It is believed that with faster operation, the stability will improve further. Recently we implemented a grid of speckles that are modulated in phase at up to $3.5 \mathrm{kHz}$. A thorough investigation of the performance of such a grid needs to be undertaken.

\subsection{High performance atmospheric dispersion compensation}

Residual atmospheric dispersion manifests as an elongation in the PSF and speckles while imaging in broadband. This results in light leaking around the coronagraphic mask which is typically optimized to suppress for an unresolved, circularly symmetric source. This in turn reduces the contrast and makes it harder to detect the close, faint companion. On ExAO systems where the wavefront errors are controlled to a high degree and result in $90 \%$ Strehl ratios in the H-band, the affect of residual atmospheric dispersion is a concern, especially for future ELT based observations.

An atmospheric dispersion compensator (ADC) typically consists of two identical prisms which can be oriented to cancel the affect of the atmosphere. The control loop for driving the prisms to the required orientation is based entirely on a model, which takes into account the telescope elevation. This indirect method of atmospheric dispersion compensation is not ideal and is limited in accuracy.

Recently we demonstrated that artificial speckles projected into the focal plane could be used as a proxy to measure the residual atmospheric dispersion. The underlying concept is that not only will the speckles be elongated, but due to atmospheric dispersion, the speckles will no longer point to the photocenter (if a line is fitted to the broadband speckles it would not intercept at the photocenter). The point of intercept is known as the radiation center. By taking some careful measurements of the affect of the ADC orientation on the separation between the photocenter and radiation center, it is possible to use the speckles to minimize this distance and reduce the residual atmospheric dispersion to $1.4 \mathrm{~ms}$ in H-band in preliminary testing. ${ }^{9,27}$ With further improvements to the control loop this will be a very useful method to reduce stellar leakage in coronagraphs on ELTs.

\subsection{Focal plane wavefront sensing}

SCExAO employs a host of other wavefront sensors including a focal plane wavefront sensor. This sensor operates at the focal plane of the internal NIR camera (see Fig. 1) nominally in the H-band. It is based on measuring the Fourier phase of a focal plane image and relating that phase to that of the wavefront in the pupil (i.e. where the DM can be used to correct it). To break the degeneracy of the problem an asymmetric mask is needed in the pupil which discards a small portion of the light. This sensor has been used on SCExAO both in the laboratory and on-sky to correct for 8 low order Zernike modes. ${ }^{10,28}$ The advantage of the sensor is that it is in the focal plane where some of the science data is collected and as such minimizes non-common path errors. It is typically used in SCExAO to correct for low order aberrations and offset the zero-point of the PyWFS to keep it well within the linear range of operation. 


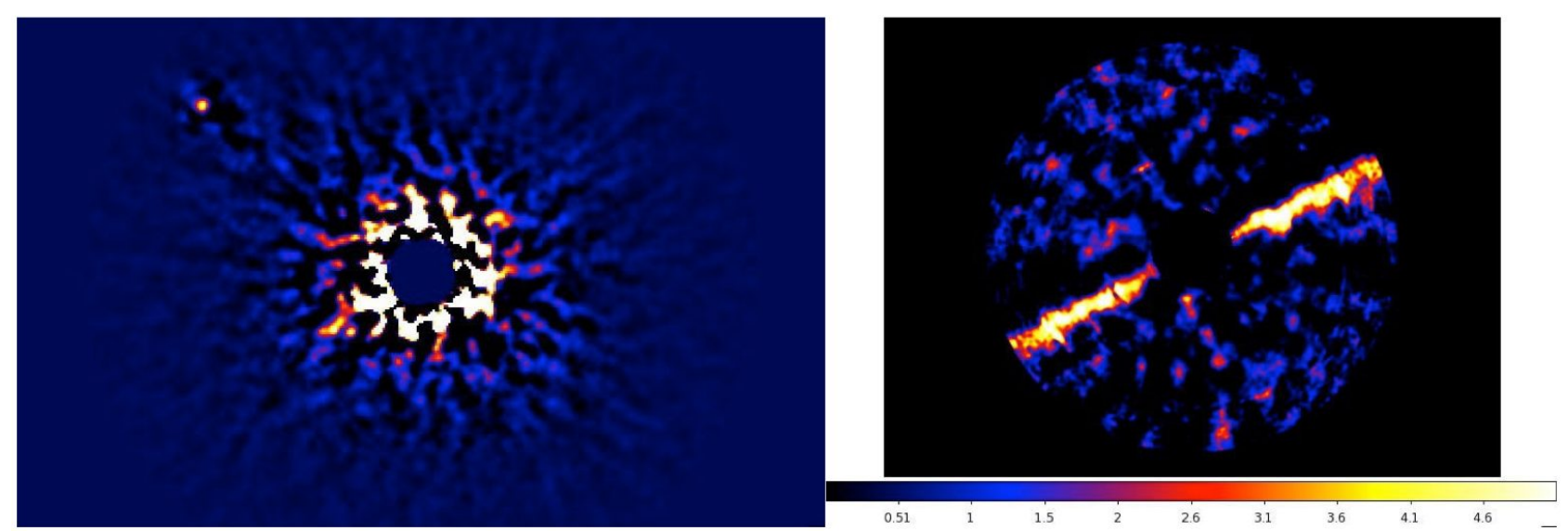

Figure 4. (Left) H-band image of Kappa Andromedae b (Right) H-band image of the disk HIP79977.

\subsection{High-contrast imaging}

With the Strehl ratio constantly improving behind SCExAO, preliminary science is now regularly under way. Figure 4 shows some examples of the data thus far. The data were acquired in the H-band. The left panel in Fig. 4 shows a reduced high-contrast image of Kappa Andromedae b and the right panel, the disk HIP79977. Both images were reduced using ALOCI/KLIP algorithms. The companion Kappa Andromedae b is clearly visible with a signal-to-noise (SNR) of 100: 1 which is a $3 \times$ improvement of the original HiCIAO only observations for a similar total integration time. In addition, the inner working angle has reduced to 300 mas also greatly improved over HiCIAO. The SNR for HIP79977 is $3-7$ a factor of $2-3 \times$ better than HiCIAO alone and the inner working angle also improved by a similar amount for a similar total integration time.

\section{SHORT TERM UPGRADES}

There are numerous short term upgrades we plan to make. These are summarized in this section.

\subsection{Post-coronagraphic, high-resolution spectroscopy}

A post-coronagraphic fiber injection is currently being developed to feed the light into the IR doppler (IRD) instrument soon to be installed at Subaru Telescope. ${ }^{29}$ This injection unit will collect light post-coronargaph where the bright light from the star has been extinguished to a high degree so that the spectrum of the planet can be detected more cleanly. This will reduce the photon-noise from the star allowing for a higher signal-to-noise spectrum of the planet to be extracted. This will offer high resolution spectroscopic insights into the chemical composition of the brightest substellar companions and has never been attempted thus far. The fiber injection has been designed and is currently under construction.

\subsection{Visible differential imaging}

The VAMPIRES module is fully operational and producing diffraction-limited images in the visible. Recently, visible imaging has received a lot of attention due to the discovery that accreting exoplanets can be directly imaging at $656 \mathrm{~nm} .{ }^{25}$ VAMPIRES will undergo a face lift which will include utilizing a faster polarization switching element, moving to a two camera system (one for each polarization) allowing for maximum imaging speeds and enabling spectral differential imaging at $\mathrm{H}$-alpha and several other wavelengths of interest. The rebuild is currently in the design phase and should be implemented by the end of 2016 .

\subsection{Nulling interferometry}

A nulling interferometer takes beams from multiple telescopes, or in this case, multiple points of a single telescope pupil and combines them interferometrically just like an aperture mask, for example. In the case of a nuller however, the aim is to look for faint objects in the dark fringe, or so-called 'null' while the object rotates onsky. This is most intuitively done in a photonic coupler/splitter. If the phase of one of the arms is carefully 
controlled at the input to the coupler it is possible to get destructive interference at one port and all the light sent to second port. The light that has been suppressed is from the host star and since the faint companion is incoherent with this, it still continues to leak out the dark port of the coupler where is can be detected. In this way, the photon-noise from the star can be reduced and the detectability of the faint companion improved. A nuller based on a 3D laser written photonic chip is currently being commissioned at Subaru. ${ }^{30}$ The system only has 2 ports thus far but upgrades to take light from numerous parts of the pupil are planned for the future. We aim to commission this module by mid-2017.

\subsection{CHARIS}

The integral field spectrograph, CHARIS, will replace the HiCIAO imager. A micro-lens array is used to section the focal plane into 'PSFlets' which are dispersed via a prism onto a H2RG detector. CHARIS will operate in a low resolution mode, $R \sim 20$, mode from $\mathrm{J}-\mathrm{K}$ band and in high resolution, $R \sim 85$, mode in any single band. ${ }^{23}$ CHARIS will provide spatially resolved spectral information that will be used to study the atmospheres of substellar companions, and differentiate the companion from speckles and background objects. It has been delivered to Subaru Telescope and is presently undergoing laboratory testing. Commissioning will begin in July and run through to the end of 2016.

\subsection{The MKID array}

SCExAO will also receive a dedicated Microwave Kinetic Inductance Detector (MKID) array. The array will consist of 20 kpixels and be optimized for efficient operation between 800-1400 nm. The MKID array will offer read-noiseless measurements at high speeds. This will be used for focal plane wavefront sensing and specifically designed to target long lived, chromatic speckle residuals. ${ }^{31}$ It is projected that it will improve the contrast by 1-2 orders of magnitude close in to the host star. The MKID array will also offer energy discrimination. Although, initially the resolving power is predicted to be of the order 2-3 in the waveband of operation, with further improvements to the technology we forecast the resolving power could be as high as 10 when complete. The MKID array will be undergo commissioning in early 2017.

\section{SUMMARY}

The SCExAO instrument is ever increasing it's arsenal of high contrast imaging technologies and capabilities. The PyWFS was recently replaced with an entirely new design that may pave the way to simpler fabrication for ELTs. RHEA could be used to study the accretion rate of forming protoplanets. CHARIS and MKIDs combined together would push the wavefront control and imaging capabilities of a high contrast imager into uncharted territory and even enable reflected light imaging of Jovian planets from Subaru Telescope. SCExAO will continue to test new and interesting technologies all the while transitioning to a platform that can produce ground-breaking scientific discoveries in routine operation.

\section{ACKNOWLEDGMENTS}

The authors acknowledge support from the JSPS (Grant-in-Aid for Research \#23340051, \#26220704 \& \#15H02063)

\section{REFERENCES}

[1] Macintosh, B., Graham, J. R., Ingraham, P., Konopacky, Q., Marois, C., Perrin, M., Poyneer, L., Bauman, B., Barman, T., Burrows, A. S., Cardwell, A., Chilcote, J., De Rosa, R. J., Dillon, D., Doyon, R., Dunn, J., Erikson, D., Fitzgerald, M. P., Gavel, D., Goodsell, S., Hartung, M., Hibon, P., Kalas, P., Larkin, J., Maire, J., Marchis, F., Marley, M. S., McBride, J., Millar-Blanchaer, M., Morzinski, K., Norton, A., Oppenheimer, B. R., Palmer, D., Patience, J., Pueyo, L., Rantakyro, F., Sadakuni, N., Saddlemyer, L., Savransky, D., Serio, A., Soummer, R., Sivaramakrishnan, A., Song, I., Thomas, S., Wallace, J. K., Wiktorowicz, S., and Wolff, S., "First light of the Gemini Planet Imager," Proceedings of the National Academy of Science 111, 12661-12666 (2014). 
[2] Vigan, A., Bonnefoy, M., Ginski, C., Beust, H., Galicher, R., Janson, M., Baudino, J.-L., Buenzli, E., Hagelberg, J., D’Orazi, V., Desidera, S., Maire, A.-L., Gratton, R., Sauvage, J.-F., Chauvin, G., Thalmann, C., Malo, L., Salter, G., Zurlo, A., Antichi, J., Baruffolo, A., Baudoz, P., Blanchard, P., Boccaletti, A., Beuzit, J.-L., Carle, M., Claudi, R., Costille, A., Delboulbé, A., Dohlen, K., Dominik, C., Feldt, M., Fusco, T., Gluck, L., Girard, J., Giro, E., Gry, C., Henning, T., Hubin, N., Hugot, E., Jaquet, M., Kasper, M., Lagrange, A.-M., Langlois, M., Le Mignant, D., Llored, M., Madec, F., Martinez, P., Mawet, D., Mesa, D., Milli, J., Mouillet, D., Moulin, T., Moutou, C., Origné, A., Pavlov, A., Perret, D., Petit, C., Pragt, J., Puget, P., Rabou, P., Rochat, S., Roelfsema, R., Salasnich, B., Schmid, H.-M., Sevin, A., Siebenmorgen, R., Smette, A., Stadler, E., Suarez, M., Turatto, M., Udry, S., Vakili, F., Wahhaj, Z., Weber, L., and Wildi, F., "First light of the VLT planet finder SPHERE. I. Detection and characterization of the substellar companion GJ 758 B," A $63 A$ 587, A55 (Mar. 2016).

[3] Dekany, R., Roberts, J., Burruss, R., Bouchez, A., Truong, T., Baranec, C., Guiwits, S., Hale, D., Angione, J., Trinh, T., Zolkower, J., Shelton, J. C., Palmer, D., Henning, J., Croner, E., Troy, M., McKenna, D., Tesch, J., Hildebrandt, S., and Milburn, J., "PALM-3000: Exoplanet Adaptive Optics for the 5 m Hale Telescope," ApJ 776, 130 (Oct. 2013).

[4] Close, L. M., Males, J. R., Morzinski, K., Kopon, D., Follette, K., Rodigas, T. J., Hinz, P., Wu, Y.-L., Puglisi, A., Esposito, S., Riccardi, A., Pinna, E., Xompero, M., Briguglio, R., Uomoto, A., and Hare, T., "Diffraction-limited Visible Light Images of Orion Trapezium Cluster with the Magellan Adaptive Secondary Adaptive Optics System (MagAO)," ApJ 774, 94 (Sept. 2013).

[5] Esposito, S., Riccardi, A., Fini, L., Puglisi, A. T., Pinna, E., Xompero, M., Briguglio, R., Quirós-Pacheco, F., Stefanini, P., Guerra, J. C., Busoni, L., Tozzi, A., Pieralli, F., Agapito, G., Brusa-Zappellini, G., Demers, R., Brynnel, J., Arcidiacono, C., and Salinari, P., "First light AO (FLAO) system for LBT: final integration, acceptance test in Europe, and preliminary on-sky commissioning results," in [Adaptive Optics Systems II], Proc. SPIE 7736, 773609 (July 2010).

[6] Jovanovic, N., Martinache, F., Guyon, O., Clergeon, C., Singh, G., Kudo, T., Garrel, V., Newman, K., Doughty, D., Lozi, J., Males, J., Minowa, Y., Hayano, Y., Takato, N., Morino, J., Kuhn, J., Serabyn, E., Norris, B., Tuthill, P., Schworer, G., Stewart, P., Close, L., Huby, E., Perrin, G., Lacour, S., Gauchet, L., Vievard, S., Murakami, N., Oshiyama, F., Baba, N., Matsuo, T., Nishikawa, J., Tamura, M., Lai, O., Marchis, F., Duchene, G., Kotani, T., and Woillez, J., "The Subaru Coronagraphic Extreme Adaptive Optics System: Enabling High-Contrast Imaging on Solar-System Scales," PASP 127, 890-910 (Sept. 2015).

[7] Lozi, J. and et. al., "High-precision wavefront control using multiple wavefront sensors: the SCExAO experience," in [Adaptive Optics Systems V], Proc. SPIE 9909, 9909181 (2016).

[8] Lozi, J. and et. al., "Characterizing and mitigating vibrations for SCExAO," in [Adaptive Optics Systems V], Proc. SPIE 9909, 990919 (2016).

[9] Pathak, P. and et. al., "First on-sky closed loop measurement and correction of atmospheric dispersion," in [Adaptive Optics Systems V], Proc. SPIE 9909, 9909196 (2016).

[10] Martinache, F. and et. al., "SCExAO: on-sky performance of the asymmetric pupil Fourier wavefront sensor," in [Adaptive Optics Systems V], Proc. SPIE 9909, 9909189 (2016).

[11] Goebel, S. and et. al., "Evolutionary timescales of AO-produced speckles at NIR wavelengths," in [Adaptive Optics Systems V], Proc. SPIE 9909, 990946 (2016).

[12] Norris, B. and et. al., "VAMPIRES: a polarimetric non-redundant-masking interferometer for diffractionlimited imaging of dusty circumstellar environments," in [Optical and Infrared Interferometry and Imaging V], Proc. SPIE 9907, 990714 (2016).

[13] Huby, E. and et. al., "Combining super-resolution and spectroscopy in the visible with FIRST at Subaru: application to binary systems and evolved stars," in [Optical and Infrared Interferometry and Imaging V], Proc. SPIE 9907, 9907104 (2016).

[14] Doughty, D. and et. al., "Current standing and upgrades to FIRST at the Subaru Telescope," in [Optical and Infrared Interferometry and Imaging V], Proc. SPIE 9907, 9907105 (2016).

[15] Rains, A. and et. al., "Precision single mode fibre integral field spectroscopy with the RHEA spectrograph," in [Ground-based and Airborne Instrumentation for Astronomy VI], Proc. SPIE 9908, 9908271 (2016).

[16] Minowa, Y., Hayano, Y., Oya, S., Watanabe, M., Hattori, M., Guyon, O., Egner, S., Saito, Y., Ito, M., Takami, H., Garrel, V., Colley, S., Golota, T., and Iye, M., "Performance of Subaru adaptive optics system AO188," in [Adaptive Optics Systems II], Proc. SPIE 7736, 77363N (July 2010). 
[17] Norris, B., Schworer, G., Tuthill, P., Jovanovic, N., Guyon, O., Stewart, P., and Martinache, F., "The VAMPIRES instrument: imaging the innermost regions of protoplanetary discs with polarimetric interferometry," MNRAS 447, 2894-2906 (Mar. 2015).

[18] Guyon, O., "Phase-induced amplitude apodization of telescope pupils for extrasolar terrestrial planet imaging," A\&̈A 404, 379-387 (June 2003).

[19] Mawet, D., Serabyn, E., Liewer, K., Burruss, R., Hickey, J., and Shemo, D., "The Vector Vortex Coronagraph: Laboratory Results and First Light at Palomar Observatory," ApJ 709, 53-57 (Jan. 2010).

[20] Kuhn, J. and et. al., "The vector vortex coronagraph observing mode on the Subaru SCExAO instrument: current status and performances," in [Ground-based and Airborne Instrumentation for Astronomy VI], Proc. SPIE 9908, 9908117 (2016).

[21] Suzuki, R., Tamura, M., Suto, H., Morino, J.-I., Hashimoto, J., Kudo, T., Kandori, R., Murakami, N., Nishikawa, J., Ukita, N., Takami, H., Guyon, O., Nishimura, T., Hayashi, M., Izumiura, H., Abe, L., Tavrov, A., Jacobson, S., Stahlberger, V., Yamada, H., Shelton, R., and Hodapp, K., "HiCIAO: A Highcontrast Instrument for the Next Generation Subaru Adaptive Optics," in [American Institute of Physics Conference Series], Usuda, T., Tamura, M., and Ishii, M., eds., American Institute of Physics Conference Series 1158, 293-298 (Aug. 2009).

[22] Brandt, T. D., McElwain, M. W., Janson, M., Knapp, G. R., Mede, K., Limbach, M. A., Groff, T., Burrows, A., Gunn, J. E., Guyon, O., Hashimoto, J., Hayashi, M., Jovanovic, N., Kasdin, N. J., Kuzuhara, M., Lupton, R. H., Martinache, F., Sorahana, S., Spiegel, D. S., Takato, N., Tamura, M., Turner, E. L., Vanderbei, R., and Wisniewski, J., "CHARIS science: performance simulations for the Subaru Telescope's third-generation of exoplanet imaging instrumentation," in [Adaptive Optics Systems IV], Proc. SPIE 9148, 914849 (July 2014).

[23] Groff, T. and et. al., "Laboratory testing and commissioning of the CHARIS integral field spectrograph," in [Ground-based and Airborne Instrumentation for Astronomy VI], Proc. SPIE 9908, 990823 (2016).

[24] Feger, T. and et. al., "Performance and future developments of the RHEA single-mode spectrograph," in [Ground-based and Airborne Instrumentation for Astronomy VI], Proc. SPIE 9908, 9908248 (2016).

[25] Sallum, S., Follette, K. B., Eisner, J. A., Close, L. M., Hinz, P., Kratter, K., Males, J., Skemer, A., Macintosh, B., Tuthill, P., Bailey, V., Defrère, D., Morzinski, K., Rodigas, T., Spalding, E., Vaz, A., and Weinberger, A. J., "Accreting protoplanets in the LkCa 15 transition disk," Nature 527, 342-344 (Nov. 2015).

[26] Jovanovic, N., Guyon, O., Martinache, F., Pathak, P., Hagelberg, J., and Kudo, T., "Artificial Incoherent Speckles Enable Precision Astrometry and Photometry in High-contrast Imaging," ApJL 813, L24 (Nov. 2015).

[27] Pathak, P., Guyon, O., Jovanovic, N., Lozi, J., Martinache, F., Minowa, Y., Kudo, T., Takami, H., Hayano, Y., and Narita, N., "First on-sky demonstration: correcting residual atmospheric dispersion for high contrast imaging," submitted to PASP (2016).

[28] Martinache, F., Jovanovic, N., and Guyon, O., "Closed-loop focal plane wavefront control with the SCExAO instrument," accepted to AछA (2016).

[29] Kotani, T., Tamura, M., Suto, H., Nishikawa, J., Sato, B., Aoki, W., Usuda, T., Kurokawa, T., Kashiwagi, K., Nishiyama, S., Ikeda, Y., Hall, D. B., Hodapp, K. W., Hashimoto, J., Morino, J.-I., Okuyama, Y., Tanaka, Y., Suzuki, S., Inoue, S., Kwon, J., Suenaga, T., Oh, D., Baba, H., Narita, N., Kokubo, E., Hayano, Y., Izumiura, H., Kambe, E., Kudo, T., Kusakabe, N., Ikoma, M., Hori, Y., Omiya, M., Genda, H., Fukui, A., Fujii, Y., Guyon, O., Harakawa, H., Hayashi, M., Hidai, M., Hirano, T., Kuzuhara, M., Machida, M., Matsuo, T., Nagata, T., Onuki, H., Ogihara, M., Takami, H., Takato, N., Takahashi, Y. H., Tachinami, C., Terada, H., Kawahara, H., and Yamamuro, T., "Infrared Doppler instrument (IRD) for the Subaru telescope to search for Earth-like planets around nearby M-dwarfs," in [Ground-based and Airborne Instrumentation for Astronomy V], Proc. SPIE 9147, 914714 (July 2014).

[30] Norris, B., Cvetojevic, N., Gross, S., Arriola, A., Tuthill, P., Withford, M., Richards, S., Goodwin, M., and Zheng, J., "An integrated-optic single-mode photonic nuller: development and on-sky testing," in [Optical and Infrared Interferometry and Imaging V], Proc. SPIE 9907, 9907119 (2016).

[31] Guyon, O., Codona, J., Fitzgerald, M., Macintosh, B., Males, J., Mawet, D., and Mazin, B., "Coronagraphic imaging of habitable exoplanets with large ground-based telescopes: fundamental limits and system architecture," in [Adaptive Optics Systems V], Proc. SPIE 9909, 990945 (2016). 